\title{
Ansiedad ante las ciencias y pruebas de evaluación científicas: efectos del nivel académico y género
}

\author{
Science and science assessment test anxiety: gender and academic level effects

\section{Ansiedade frente às ciências e testes de avaliação científicos: efeitos do nível acadêmico e género}

\author{
Marc Benlloch Pla ${ }^{1}$ \\ Joan Josep Solaz Portolés ${ }^{2}$ \\ Vicent Sanjosé López ${ }^{3}$
}

Recibido: junio de 2018

Aceptado: diciembre de 2018

Para citar este artículo: Benlloch-Pla, M., Solaz-Portolés, J. J. Y Sanjosé-López, V. (2019). Ansiedad ante las ciencias y pruebas de evaluación científicas: efectos del nivel académico y genero. Revista Científica, 34(1), 20-33. Doi: https://doi.org/10.14483/23448350.13542

\section{Resumen}

En este trabajo se hace una primera aproximación a la ansiedad de los estudiantes de secundaria ante las ciencias. Se han aplicado a 175 estudiantes de educación secundaria de diferentes cursos dos cuestionarios validados y una prueba de evaluación de competencias científicas. Un cuestionario mide la ansiedad ante diversos contextos científicos y el otro evalúa la ansiedad generada por una prueba de evaluación de ciencias. El análisis de los resultados obtenidos por medio de pruebas Anova evidencia que: a) la ansiedad ante situaciones científicas es mayor que en otras situaciones y disminuye significativamente con el aumento del nivel académico; b) la ansiedad generada en la prueba de evaluación es mayor, de forma estadísticamente significativa, en las chicas que en los chicos; y c) no se ha constatado una correlación significativa entre el nivel de ansiedad generada por la prueba de evaluación y el rendimiento conseguido en esta prueba.

Palabras clave: ansiedad ante las ciencias, educación secundaria, género, nivel académico, prueba de evaluación científica.

\begin{abstract}
In this paper is carried out a first approximation to anxiety surrounding situations related to science. One hundred and seventy-five spanish secondary education students at different educational levels have been administered two validated tests and a scientific competencies assessment test. One test measures the anxiety caused by scientific contexts, and the other test assesses the science assessment test anxiety. The analysis of the results obtained by means of Anova tests allow us to conclude that: a) the anxiety in scientific situations is greater than
\end{abstract}


the non-scientific situations, and this anxiety gets smaller as the school grade level increases. ; b) the science assessment test anxiety is statistically significantly higher for women than for men; and c) there is no evidence of a significant correlation between the science assessment test anxiety and science test scores.

Keywords: science anxiety, secondary education, gender, school grade level, science assessment test.

\section{Resumo}

Uma primeira aproximação é feita à ansiedade dos estudantes de secundário frente às ciências neste trabaIho. Foram administrados dois questionários validados e um teste de avaliação de competências científicas a 175 estudantes de educação secundária de cursos diferentes. Um questionário mede a ansiedade frente à diferentes contextos científicos e o outro avalia a ansiedade gerada por um teste de avaliação das ciências. A análise dos resultados obtidos por meio de testes Anova mostra que: a) a ansiedade frente à situações científicas é maior que em outras situações, e diminui significativamente com o aumento do nível acadêmico; b) a ansiedade gerada no teste de avaliação é maior, de modo estatisticamente significante, nas raparigas que nos rapazes; e c) não foi confirmada uma correlação significante entre o nível de ansiedade gerado pelo teste de avaliação e a pontuação obtida neste teste.

Palavras-chaves: ansiedade frente às ciências, ensino secundário, género, nível acadêmico, teste de avaliação das ciências.

\section{Introducción}

Las reacciones afectivas o emocionales ante una tarea de aprendizaje constituyen un componente relevante de la motivación (Pintrich y De Groot, 1990). En las situaciones de aprendizaje en la escuela, una de las reacciones emocionales más importantes parece ser la denominada test anxiety o ansiedad ante las pruebas de evaluación (Wigfield y Eccles, 1989). Se trata de un tipo de ansiedad que se manifiesta en el contexto de la evaluación y de la cual existe un abundante cuerpo de literatura especializada que ha establecido sus orígenes, correlaciones y tratamientos (Putwain, 2007). Los resultados de la investigación llevada a cabo por Matthews, Hillyard y CampbeII (1999) muestran que la test anxiety está relacionada con dos factores cognitivos diferentes: uno ligado a la metacognición y preocupación, y el otro vinculado a un estilo inapropiado de afrontar las circunstancias.

En un artículo de Hembree (1988) se destaca que los niveles altos de ansiedad ante pruebas de evaluación tienen una influencia negativa en el rendimiento académico en diversas asignaturas y niveles académicos. Según Cassady y Johnson (2002), existe una amplia aceptación de que la ansiedad ante pruebas de evaluación tiene dos dimensiones: la emocional (que se evidencia a través de respuestas fisiológicas) y la cognitiva (asociada a creencias autodespectivas de la propia capacidad que se manifiestan, por ejemplo, en la ideas de los sujetos sobre las consecuencias del fracaso, bajo nivel de autoestima, excesiva preocupación por la evaluación, miedo a la reacción de los padres, etc.). Por otra parte, se ha comprobado que las estudiantes presentan niveles de ansiedad ante las pruebas de evaluación significativamente más altos que los estudiantes (Hembree, 1988; Zeidner, 1998). Además, se ha puesto en evidencia que estas diferencias de género son más grandes en la componente emocional (Zeidner, 1990; Zeidner y Schleyer, 1999).

Las matemáticas son una de las disciplinas académicas en la que con más prontitud se investigaron los efectos de la ansiedad y, además, en la que se ha realizado mayor número de investigaciones (Ashcraft, 2002; Ashcraft y Krause, 2007; Ashcraft y Moore, 2009; Wigfield y Meece, 1988). En todos estos trabajos se habla de ansiedad matemática o ansiedad ante las matemáticas (math anxiety). Los primeros autores que utilizaron este término definieron la ansiedad matemática como los sentimientos de tensión y desasosiego que interfieren las operaciones o la resolución de problemas de matemáticas en una amplia variedad de situaciones (Richardson y Suinn, 1972). 
El concepto de ansiedad científica o ansiedad ante la ciencia (science anxiety) fue acuñado por Mallow (1978) en la década de 1970. Fue definido por las investigadoras Wynstra y Cummings (1990, p. 1) como "un temor difuso o vago que se genera en respuesta ante situaciones relacionadas con el aprendizaje de la ciencia". Según Mallow y Greenburg (1983), esta ansiedad es el resultado de creencias propias y no del aprendizaje de las ciencias en sí mismo. Las causas de la ansiedad científica pueden ser muchas: malas experiencias previas en las clases de ciencias, haber tenido maestros a los que las ciencias les generaba ansiedad, ausencia de modelos a seguir, estereotipos de género o la preeminencia en los medios de comunicación de un determinado estereotipo del científico (Mallow, 2006). No obstante, un cierto nivel de ansiedad puede incluso llegar a ser provechoso en los procesos de aprendizaje de las ciencias (Mallow y Greenburg, 1982). En la práctica, la ansiedad científica actúa como filtro en la selección de los estudios posteriores, ya que hace que los estudiantes sientan temor de las disciplinas científicas y no las cursen (Udo, Ramsey y Mallow, 2004).

Desde la década de 1980 se está investigando la influencia de la ansiedad científica en las diferencias de género observadas en el aprendizaje de las ciencias (Beyer, 1991; Chiarelott y Czerniak, 1987; Mallow, 1987). Concretamente, en el estudio de Chiarelott y Czerniak (1987) se observaron diferencias de ansiedad científica entre niños y niñas desde los nueve años, porcentajes mayores de niñas con alta ansiedad y una correlación negativa entre el nivel de ansiedad y el rendimiento académico (mayor nivel de ansiedad menor rendimento académico).

Mallow (1994), en una investigación con estudiantes americanos y daneses mayores de 17 años en la que utilizó un cuestionario que autovaloraban su ansiedad, mostró que las mujeres declaraban niveles de ansiedad significativamente más altos que los hombres. Udo, Ramsey, Reynolds-Alpert y Mallow (2001) encontraron que tras un curso de Física universitaria se reducía la ansiedad científica, aunque siempre los niveles de ansiedad eran superiores en las chicas. Los estudios realizados por Udo et al. (2004) con universitarios; y los de Bryant, Kastrup, Udo, Hislop, Shefnery MaIlow, J. (2013) con estudiantes de últimos cursos de secundaria y universitarios confirmaron nuevamente que las mujeres generan una mayor ansiedad ante las ciencias. Las investigadoras Wynstra y Cummings (1990), en un trabajo realizado con estudiantes de los últimos cursos de High School, además de volver a hallar mayor ansiedad en las chicas que en los chicos, encontraron que el nivel de ansiedad científica disminuía con los años que se cursaban de Química. Así, los estudiantes de grado 12 que habían cursado más años la asignatura de Química eran los que menos ansiedad hacia la ciencia presentaban.

En Latinoamérica es de destacar el estudio realizado por Quintero, Pérez y Correa (2009) con tres grupos de estudiantes mexicanos de educación secundaria de distintos niveles académicos. En este estudio se examinó la relación entre la autoeficacia y la ansiedad ante las ciencias; como resultado se encontró una correlación negativa y estadísticamente significativa entre ellas. En España no se ha efectuado todavía ninguna investigación sobre la ansiedad ante las ciencias entre los estudiantes de educación secundaria, pero sí ante las matemáticas (Muñoz y Mato, 2007). El presente estudio es, precisamente, una primera aproximación a esta cuestión. Pretende analizar el nivel de ansiedad de los estudiantes de secundaria ante las ciencias en general y, en particular, la asociada a la realización de una prueba/actividad académica de ciencias.

\section{Marco teórico}

Se ha señalado anteriormente que hay trabajos, realizados sobre todo en Estados Unidos de América, que muestran que: la ansiedad de los estudiantes suele ser superior en contextos científicos que en los no científicos; esta ansiedad suele ser 
mayor en las chicas que en los chicos; la ansiedad ante las ciencias disminuye a medida que aumentan los conocimientos científicos; y una mayor ansiedad conduce a un menor rendimiento académico. No hay razones para creer que en un ámbito territorial diferente estos resultados sean diferentes, por tanto, se formulan las siguientes hipótesis referidas a estudiantes de secundaria valencianos (España):

1. La ansiedad de los estudiantes en contextos científicos será superior que en la de contextos no científicos.

2. La ansiedad ante las ciencias disminuirá significativamente con el nivel académico.

3. El nivel de ansiedad ante las ciencias será significativamente mayor en las chicas que en los chicos.

4. Existirá una correlación inversa y significativa entre el nivel de ansiedad generada por una prueba de evaluación de competencias científicas y el rendimiento conseguido en esta prueba.

\section{Metodología}

\section{Diseño experimental}

Se trata de un diseño transversal descriptivo. Las variables independientes son el nivel académico y el género. Las variables dependientes son el nivel de competencia científica (medido con una prueba de evaluación: una prueba Pisa), la ansiedad científica (ansiedad en contextos científicos), la ansiedad no científica (ansiedad en contextos no científicos) y la ansiedad generada por una prueba de evaluación de competencias científicas (prueba Pisa).

\section{Sujetos participantes}

En el estudio han participado 175 estudiantes (89 chicas y 86 chicos) de un centro educativo público de educación secundaria del País Valenciano (España), de los cuales 147 son de educación secundaria obligatoria $(\mathrm{ESO})^{4}$, y 28 son de bachiIlerato científico-tecnológico ${ }^{5}$ (. Se trata de una muestra de conveniencia $y$, consecuentemente, los resultados y las conclusiones que se obtengan solo pueden ser válidas stricto sensu para los sujetos que han intervenido en el estudio.

El número de alumnos en cada nivel académico aparece en la tabla 1.

Tabla 1. Número de estudiantes participantes por nivel académico.

$\begin{array}{cc}\text { Nivel académico } & \text { Número de estudiantes } \\ 2^{\circ} \text { ESO (13 años) } & 49 \\ 3^{\circ} \text { ESO (14 años) } & 45 \\ 4^{\circ} \text { ESO (15 años) } & 53 \\ 1^{\circ} \text { bachillerato (16 años) } & 28\end{array}$

Fuente: elaboración propia de los autores.

\section{Instrumentos}

En primer lugar, para medir la ansiedad científica se ha usado un cuestionario validado y ampliamente utilizado en diversas investigaciones (Udo et al., 2004). En realidad, este cuestionario registra la ansiedad tanto en contextos científicos como en contextos no científicos. En el anexo 1 puede verse la versión castellana y adaptada de dicho instrumento. Se compone de 44 ítems que exponen diferentes situaciones y experiencias que pueden causar temor o angustia. La mitad de estas situaciones se desarrollan en un contexto científico, mientras que la otra mitad no. Además, los ítems se encuentran emparejados y exponen situaciones análogas que en un caso se contextualizan en el ámbito científico y en el otro en un contexto cotidiano.

El cuestionario empleado para determinar la ansiedad generada por una prueba de evaluación

\footnotetext{
Período obligatorio de escolarización en España de cuatro cursos que va desde los 11-12 a los 15-16 años; en el $4^{\circ}$ curso de la ESO los estudiantes pueden elegir entre un itinerario científico-tecnológico o uno humanístico-ciencias sociales.

5. Período educativo no obligatorio que va de los 16-17 a los 17-18 años y que prepara para el acceso a la universidad.
} 
de competencia científica ha sido el GTAI-A (Heredia, Piemontesi, Furlan y Volker, 2008). Dicho cuestionario permite medir la tendencia estable de los individuos de sufrir ansiedad ante situaciones de carácter evaluativo. Incluye 28 ítems que analizan diferentes sentimientos o sensaciones que el alumno ha podido sentir durante una prueba de evaluación realizada previamente. Estos ítems valoran cuatro niveles diferentes de la ansiedad: emocionalitad ("me he sentido nervioso/osa"), preocupación ("pienso en las consecuencias de fracasar"), falta de confianza ("sé que puedo confiar en mí mismo") e interferencia en los pensamientos ("de repente me cruzan pensamientos por la cabeza que me bloquean").

Finalmente, se ha utilizado una prueba Pisa (Ministerio de Educación, 2010) para medir las competencias científicas de los alumnos. Este tipo de pruebas han sido elaboradas para que los alumnos demuestren si tienen una serie de competencias, es decir, permiten ver si los estudiantes son capaces de resolver un problema práctico aplicando determinados conocimientos y mediante el pensamiento crítico. Las pruebas huyen, por lo tanto, de la memorización y buscan la racionalidad como clave para ser superadas. La prueba Pisa elegida consta de tres preguntas que miden la capacidad para explicar fenómenos de forma científica. Analizan, además, si los alumnos poseen conocimientos sobre la investigación científica, sistemas físicos (mezclas) y química básica.

\section{Procedimiento}

En una primera sesión se repartió la prueba Pisa para evaluar las competencias científicas. A los estudiantes se les informó que con la prueba Pisa se quería determinar su nivel de competencias científicas. Inmediatamente después se administró el cuestionario que mide la ansiedad ante una prueba de evaluación. En una segunda sesión se administró el cuestionario que mide la ansiedad hacia las ciencias. Se pidió a los alumnos que en caso de no haber experimentado la situación planteada de algún ítem la imaginaran.

Cada una de las tres preguntas de la prueba Pisa se puntuó siguiendo los criterios que se establecen en las mismas pruebas (Ministerio de Educación, 2010), con un punto o cero puntos, según si estaba contestada correcta o incorrectamente. Por lo tanto, en cada prueba se obtenía para cada estudiante una puntuación comprendida entre cero y tres puntos. La primera pregunta es de respuesta abierta, la segunda y tercera son preguntas de opción múltiple (cuatro opciones y una sola respuesta correcta).

El cuestionario para evaluar la ansiedad científica utiliza una escala tipo Likert de cinco niveles de respuesta "nada", "muy poco", "un poco", "bastante", o "mucho". Las respuestas fueron puntuadas con una escala de uno ("nada") a cinco ("mucho"). Además, las preguntas se agruparon en dos grupos o factores, según si pertenecían a un contexto científico o no. Así pues, de cada cuestionario se obtuvo una media aritmética entre uno (poca ansiedad) y cinco (mucha ansiedad) para situaciones en un contexto científico y otra para situaciones sin contexto científico.

El cuestionario para determinar la ansiedad generada por una prueba de evaluación de competencias científicas emplea también una escala tipo Likert, pero de cuatro niveles, que van desde "nada" a "mucho", según la ansiedad producida. En el primer caso se le otorga un punto y en el último cuatro. No obstante, como los ítems 1, 4, 8, 13,20 y 26 de dicho cuestionario estaban formulados de forma contraria, su puntuación se invirtió antes de obtener la media para cada cuestionario.

\section{Resultados}

En cuanto a la fiabilidad de los cuestionarios, el coeficiente alfa de Cronbach en el cuestionario para medir la ansiedad ante las ciencias es de 0.87 en los ítems de ansiedad ante las ciencias, y de 0.88 en los ítems de ansiedad en contextos no científicos. En el caso del cuestionario de ansiedad 
generada por la prueba de evaluación, el valor de este coeficiente es de 0.84. Así pues, podemos considerar que la fiabilidad de los cuestionarios es alta. Por otro lado, el test de Kolmogorov-Smirnov aplicado a las puntuaciones de los cuestionarios en cada nivel académico conduce a valores los niveles de significación que siempre están por encima de .05. Por lo tanto, en todos los casos se puede rechazar la hipótesis nula y puede considerarse que todas las puntuaciones siguen una distribución normal.

La figura 1 nos ofrece la media aritmética de las puntuaciones de los estudiantes en el cuestionario para medir la ansiedad ante las ciencias en cada nivel académico. En concreto, presenta tanto la puntuación media en los ítems que miden el nivel de ansiedad ante las ciencias, como en los ítems que miden la ansiedad en contextos no científicos.

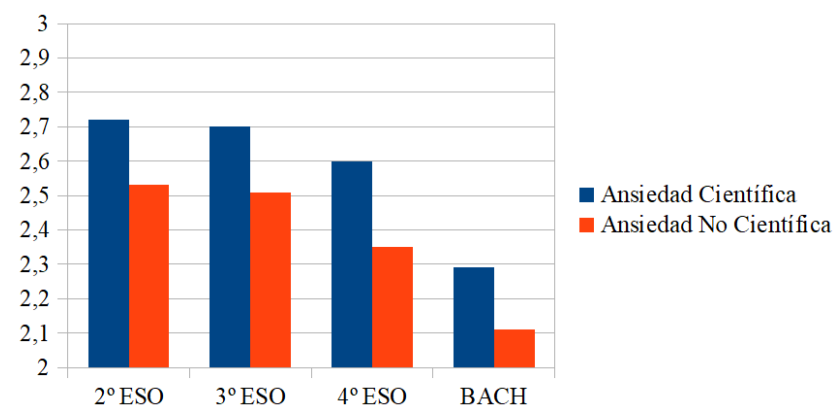

Figura 1. Puntuaciones medias de los distintos niveles académicos en los dos factores del cuestionario de media de ansiedad ante las ciencias.

Fuente: elaboración propia de los autores.

Se llevó a cabo una análisis de varianza (Anova) tomando como variable intrasujetos el tipo de ansiedad (con dos valores, ansiedad científica y ansiedad no científica), como variable intersujetos nivel académico (con cuatro niveles: $2^{\circ}, 3^{\circ}$ y $4^{\circ}$ de ESO y bachillerato), y como variable dependiente la puntuación media de los ítems correspondientes. Los resultados de este Anova revelan que: a) el nivel de ansiedad ante situaciones científicas es significativamente superior que ante situaciones no científicas (con un tamaño del efecto pequeño), $F(1,167)=10.74, p<.01, \eta 2=.03 ; b)$ un mayor nivel académico reduce significativamente el nivel de ansiedad (con un tamaño del efecto medio), $\mathrm{F}(3,167)=7.43, \mathrm{p}<.001, \mathrm{\eta} 2=.06$; y c) la interacción entre tipo de ansiedad y nivel académico no produce efectos significativos. Además, la aplicación de pruebas post hoc evidencia diferencias significativas de puntuación en el nivel de ansiedad científica entre $2^{\circ}$ y $4^{\circ}$ de la ESO $(p<.05)$, entre $4^{\circ}$ de la ESO y bachillerato $(p<.01)$, y entre $2^{\circ}$ y $3^{\circ}$ de ESO y bachillerato $(p<.01)$.

En la figura 2 aparecen las puntuaciones medias de ansiedad científica según el género y el nivel académico de los estudiantes. En la figura 3 se representan las puntuaciones medias de la ansiedad generada en chicos y chicas por la prueba de evaluación de competencias científicas en los distintos niveles académicos.

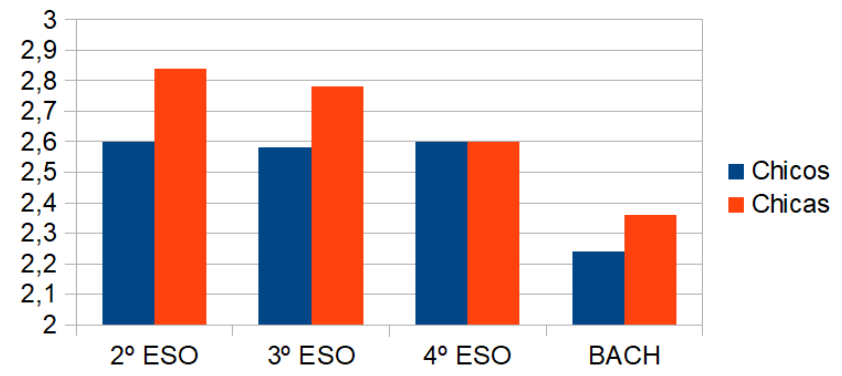

Figura 2. Representación gráfica de las puntuaciones medias de ansiedad científica en función del nivel académico y del género de los estudiantes.

Fuente: elaboración propia de los autores.

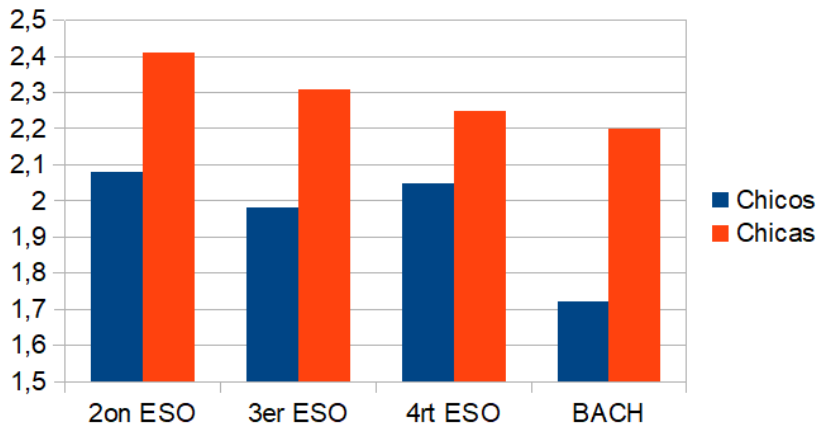

Figura 3. Representación gráfica de las puntuaciones medias de ansiedad generada por una prueba de evaluación de competencias científicas, en función del nivel académico y del género de los estudiantes.

Fuente: elaboración propia de los autores. 
Se hicieron dos análisis de varianza. En ambos se tomaron como variables intersujetos el género (con dos valores, chica y chico) y el nivel académico (con cuatro niveles: $2^{\circ}, 3^{\circ}$ y $4^{\circ}$ de ESO y bachillerato). En el primer Anova la variable dependiente fue la puntuación media de los ítems de ansiedad científica. En el segundo Anova la variable dependiente fue la puntuación media de los ítems de la prueba que mide la ansiedad originada por una prueba de evaluación de competencias científicas. Los resultados del primer Anova revelan que: a) el género no llega a produir diferencias significativas en el nivel de ansiedad científica ( $p=.06) ; b)$ el nivel académico reduce significativamente el nivel de ansiedad científica (con un tamaño del efecto medio), $\mathrm{F}(3,167)=3.50, \mathrm{p}<.05, \eta 2=.06 ;$ y c) la interacción entre sexo y nivel académico no produce efectos significativos. Los resultados del segundo Anova ponen de manifiesto que: a) las chicas generan una ansiedad por la prueba significativamente mayor que los chicos (con un tamaño del efecto medio-alto), $F(1,167)=24.98, p<.001$, $\eta 2=.12 ; b)$ el nivel académico reduce significativamente el nivel de ansiedad generada por la prueba (con un tamaño del efecto medio), $\mathrm{F}(3,167)=3.90, \mathrm{p}<.05, \eta 2=.06 ;$ y c) la interacción entre género y nivel académico no produce efectos significativos.
En la tabla 2 se recogen los coeficientes de correlación lineal producto-momento de Pearson entre la puntuaciones del nivel de ansiedad científica, de la prueba de evaluación de competencias científicas y del nivel de ansiedad generado en dicha prueba de evaluación.

\section{Discusión y conclusiones}

En primer lugar, se tiene que hacer referencia a las limitaciones de este estudio. La principal limitación se deriva de la naturaleza y el tamaño de la muestra empleada. Los resultados y las conclusiones que hemos obtenido solamente pueden ser válidos stricto sensu para los sujetos que han intervenido en ella.

Como puede verse en la figura 1, el nivel de ansiedad es medio-bajo (entre 2 y 3, en un a escala de 1 a 5) y más alto siempre en el caso de la ansiedad cientifica. Este resultado es coherente con el obtenido por Quintero et al. (2009). Además, se hace sentir el efecto del nivel académico claramente a partir de $4^{\circ}$ de la ESO. Debe tenerse en cuenta que en nuestra muestra de estudiantes un mayor nivel académico lleva asociado haber cursado más asignaturas científicas, esto es, mayores conocimientos científicos. Por otro lado, el Anova efectuado ha mostrado que el nivel de ansiedad en contextos científicos es significativamente superior

Tabla 2. Coeficientes de correlación entre las puntuaciones de las tres medidas realizadas en cada nivel académico.

\begin{tabular}{|c|c|c|c|c|}
\hline & \multicolumn{2}{|c|}{$\begin{array}{c}\text { Ansiedad cientifica vs. prueba } \\
\text { evaluación }\end{array}$} & \multicolumn{2}{|c|}{$\begin{array}{c}\text { Ansiedad generada vs. prueba } \\
\text { evaluación }\end{array}$} \\
\hline & $\begin{array}{c}\text { Coef. de } \\
\text { correlación }\end{array}$ & $p$ & $\begin{array}{c}\text { Coef. de } \\
\text { correlación }\end{array}$ & $p$ \\
\hline $\begin{array}{c}2^{\circ} \mathrm{ESO} \\
(\mathrm{g} . \mathrm{I}=47)\end{array}$ & -0.24 & 0,10 & -0.15 & 0.31 \\
\hline $\begin{array}{l}3^{\circ} \mathrm{ESO} \\
(\mathrm{g} . \mathrm{I}=43)\end{array}$ & -0.23 & 0.13 & 0.01 & 0.95 \\
\hline $\begin{array}{c}4^{\circ} \mathrm{ESO} \\
(\text { g.l. }=51)\end{array}$ & 0.06 & 0.67 & 0.11 & 0.43 \\
\hline $\begin{array}{c}\text { Bachillerato } \\
(\text { g.l. }=26)\end{array}$ & 0.11 & 0.58 & 0.02 & 0.92 \\
\hline
\end{tabular}

Ansiedad científica vs. ansiedad generada Coef. de correlación $\quad p$ $0.47<0.001$

$0.33 \quad 0.03$

$0.51<0.001$

$\begin{array}{ll}0.47 & 0.01\end{array}$

Fuente: elaboración propia de los autores. 
que ante contextos no científicos, lo que nos permite confirmar la primera hipótesis planteada.

Las figuras 1, 2 y 3 subrayan la importancia del nivel académico (conocimientos científicos) en la reducción de la ansiedad, tanto en contextos científicos como en las pruebas de evaluación de competencias científicas. Los Anovas realizados muestran también que la variable nivel académico genera reducciones significativas en ambos tipos de ansiedad. Todo ello está en linea con el trabajo de las investigadoras Wynstra y Cummings (1990) y valida la formulación de la segunda hipótesis.

Los efectos de la variable género pueden observarse en las figuras 2 y 3 . Los niveles de ansiedad científica y de ansiedad en las pruebas de evaluación de competencias científicas de las chicas siempre están por encima de los chicos (salvo en un caso, que son iguales). Este hecho ha quedado reflejado sobre todo en el caso de la ansiedad en las pruebas de evaluación de competencias científicas en las cuales el correspondiente Anova ha puesto de relieve los efectos significativos de la variable género. Este resultado es acorde con los estudios de Udo et al. (2001), Udo et al. (2004) y Bryant et al. (2013) y matiza el planteamiento la hipótesis tercera. En concreto, solo le otorga validez ante pruebas de evaluación de competencias científicas. Esto es, se ha constatado que las chicas generan un nivel de ansiedad estadísticamente más elevado que los chicos unicamente en el caso de pruebas de evaluación de competencias científicas.

De los coeficientes de correlación que aparecen en la tabla 2, parece colegirse que solo en los niveles académicos inferiores apareceren correlaciones negativas bajas entre ansiedad científica o ansiedad ante pruebas de evaluación de competencias científicas y las puntuaciones obtenidas en dichas pruebas. A medida que el nivel académico aumenta, dichas correlaciones se hacen positivas, aunque en ningún nivel académico llegan a ser estadísticamente significativas. Por tanto, esto conduce a refutar la cuarta hipótesis de este trabajo. Es decir, no se ha confirmado que una mayor ansiedad ante las ciencias conduzca a un menor rendimiento en pruebas de evaluación de ciencias. Esto entra en contradicción con los resultados del estudio de Chiarelott y Czerniak (1987), si bien los estudiantes que participaron en él eran de una edad bastante menor.

No se pueden dejar de destacar los valores de los coeficientes de correlación entre los dos niveles de ansiedad medidos en el presente estudio y que figuran en la tabla 2. Todos son positivos, moderados y significativos. Ello viene a confirmar que, en realidad, los instrumentos que se han utilizado estan midiendo el mismo constructo; a este se le podría denominar genéricamente "ansiedad ante las ciencias".

\section{Referencias}

Ashcraft, M. H. (2002). Math anxiety: Personal, educational, and cognitive consequences. Current Directions in Psychological Science, 11(5), 181-185. DOI: https://doi. org/10.1111/1467-8721.00196

Ashcraft, M. H. y Krause, J. A. (2007). Working memory, math performance, and math anxiety. Psychonomic Bulletin y Review, 14(2), 243-248. DOI: https://doi.org/10.3758/ BF03194059

Ashcraft, M. H. y Moore, A. M. (2009). Mathematics anxiety and the affective drop in performance. Journal of Psychoeducational Assessment, 27(3), 197-205. DOI: https://doi. org/10.1177/0734282908330580

Beyer, K. (1991). Gender, science, anxiety and learning styles. En: L. J. Rennie, L. H. Parker y G. M. Hildebrand (eds.), Action for equity: The second decade (pp. 10-17). Perth: National Key Centre for Teaching and Research in School Science and Mathematics.

Bryant, F. B., Kastrup, H., Udo, M., Hislop, N., Shefner, R. y Mallow, J. (2013). Science anxiety, science attitudes, and constructivism: A binational study. Journal of Science Education 
and Technology, 22(4), 432-448. DOI: https:// doi.org/10.1007/s10956-012-9404-x

Cassady, J. C. y Johnson, R. E. (2002). Cognitive test anxiety and academic performance. Contemporary Educational Psychology, 27(2), 270-295. DOI: https://doi.org/10.1006/ ceps.2001.1094

Chiarelott, L. y Czerniak, C. (1987). Speaking Out: Science Anxiety: Implications for Science Curriculum and Teaching. The Clearing House, 60(5), 202-205. DOI: https://doi.org/10.1080/ $\underline{00098655.1987 .9959323}$

Hembree, R. (1988). Correlates, causes, effects, and treatment of test anxiety. Review of Educational Research, 58(1), 47-77. DOI: https://doi. org/10.3102/00346543058001047

Heredia, D., Piemontesi, S., Furlan, L. y Volker, H. (2008). Adaptación del inventario alemán de ansiedad frente a los exámenes: GTAI-A. Revista Evaluar, 8, 46-60.

Mallow, J. V. (1978). A science anxiety program. American Journal of Physics, 46(8), 862-862. DOI: https://doi.org/10.1119/1.11409

Mallow, J. V. (1987). Science anxiety and gender. Bulletin of Science, Technology y Society, 7(3-4), 958-962. DOI: https://doi. org/10.1177/027046768700700389

Mallow, J. V. (1994). Gender-related science anxiety: A first binational study. Journal of Science Education and Technology, 3(4), 227-238. DOI: https://doi.org/10.1007/BF01575898

Mallow, J. V. (2006). Science anxiety: research and action. En J. J.Mintzes y W. H. Leonard (eds.), Handbook of College Science Teaching (pp. 325-349). Arlington, EE.UU.: NSTA Press.

Mallow, J. V. y Greenburg, S. L. (1982). Science anxiety: Causes and remedies. Journal of $\mathrm{CO}^{-}$ llege Science Teaching, 356-358.

Mallow, J. V. y Greenburg, S. L. (1983). Science anxiety and science learning. The Physics Teacher, 21(2), 95-99. DOI: https://doi. org/10.1119/1.2341214

Matthews, G., Hillyard, E. J. y Campbell, S. E. (1999). Metacognition and maladaptive coping as components of test anxiety. Clinical Psychology y Psychotherapy, 6(2), 111-125. DOI: https://doi.org/10.1002/(SICI)1099-0879(19990 5)6:2<111::AID-CPP192>3.0.CO;2-4

Ministerio de Educación (2010). Ciencias en Pisa. Pruebas liberadas. Madrid: Ministerio de Educación. Recuperado de https://www.academia.edu/7075799/Ciencias-en-pisa-para-web

Muñoz, J. M. y Mato, M. D. (2007). Elaboración y estructura factorial de un cuestionario para medir la "ansiedad hacia las matemáticas" en alumnos de educación secundaria obligatoria. Revista Galego-Portuguesa de Psicoloxía e Educación, 14, 221-234.

Pintrich, P. R. y De Groot, E. V. (1990). Motivational and self-regulated learning components of classroom academic performance. Journal of Educational Psychology, 82(1), 33-40. DOI: https://doi.org/10.1037/0022-0663.82.1.33

Putwain, D. W. (2007). Test anxiety in UK schoolchildren: Prevalence and demographic patterns. British Journal of Educational Psychology, 77(3), 579-593. DOI: https://doi. org/10.1348/000709906X161704

Quintero, M. A., Pérez, E. y Correa, S. (2009). La relación entre la autoeficacia y la ansiedad ante las ciencias en estudiantes del nivel medio superior. Revista Internacional de Ciencias Sociales y Humanidades, 19(2), 69-91.

Richardson, F. C. y Suinn, R. M. (1972). The mathematics anxiety rating scale: Psychometric data. Journal of Counseling Psychology, 19(6), 551-554. DOI: https://doi.org/10.1037/ $\underline{\mathrm{h} 0033456}$

Udo, M. K., Ramsey, G. P. y Mallow, J. V. (2004). Science anxiety and gender in students taking general education science courses. Journal of Science Education and Technology, 13(4), 435-446. DOI: https://doi.org/10.1007/ s10956-004-1465-Z

Udo, M. K., Ramsey, G. P., Reynolds-Alpert, S. y Mallow, J. V. (2001). Does physics teaching affect gender-based science anxiety? Journal of Science Education and 
Technology, 10(3), 237-247. DOI: https://doi. org/10.1023/A:1016686532654

Wigfield, A. y Meece, J. L. (1988). Math anxiety in elementary and secondary school students. Journal of Educational Psychology, 80(2), 210-216. DOI: https://doi. org/10.1037/0022-0663.80.2.210

Wigfield, A. y Eccles, J. S. (1989). Test anxiety in elementary and secondary school students. Educational Psychologist, 24(2), 159-183. DOI: https://doi.org/10.1207/s15326985ep2402 3

Wynstra, S. y Cummings, C. (1990). Science Anxiety: Relation with Gender, Year in Chemistry Class, Achievement, and Test Anxiety. Ponencia presentada en Annual Meeting of the Midwestern Educational Rtsearch Association (Chicago, EE.UU., octubre 17-19). Recuperado de https://files.eric.ed.gov/fulltext/ ED331837.pdf

Zeidner, M. (1990). Does test anxiety bias scholastic aptitude test performance by gender and sociocultural group? Journal of Personality Assessment, 55(1-2), 145-160. DOI: https://doi. org/10.1080/00223891.1990.9674054

Zeidner, M. (1998). Test anxiety: The state of the art. Nueva York: Kluwer Academic Publishers.

Zeidner, M. y Schleyer, E. J. (1999). Test anxiety in intellectually gifted school students. Anxiety, Stress and Coping, 12(2), 163-189. DOI: https://doi.org/10.1080/10615809908248328 


\section{Anexo I}

\section{Cuestionario de ansiedad ante las Ciencias}

(Traducido y adaptado de Udo, Ramsey y Mallow, 2004)

Nombre:

Curso:

Los siguientes ítems explican situaciones y experiencias que pueden causar temor o aprensión. Para cada uno de ellos, rodea el grado de temor, turbación o desasosiego que te produce.

1. Aprender cómo convertir grados Celsius a Fahrenheit para viajar a otros países

$$
\text { Nada Muy poco Un poco Bastante Mucho }
$$

2. En un debate en una clase de Historia, exponer un tema sobre la corona de Aragón y tener que responder a unas preguntas

$$
\text { Nada Muy poco Un poco Bastante Mucho }
$$

3. Responder una pregunta en clase de Física, Química o Biología

$$
\text { Nada Muy poco Un poco Bastante Mucho }
$$

4. Convertir las hectáreas en metros cuadrados

$$
\text { Nada Muy poco Un poco Bastante Mucho }
$$

5. Estudiar para un examen de química, física o biología

$$
\text { Nada Muy poco Un poco Bastante Mucho }
$$

6. Planificar una dieta sana y equilibrada

$$
\text { Nada Muy poco Un poco Bastante Mucho }
$$

7. Convertir los euros a libras inglesas para viajar a Inglaterra

$$
\text { Nada Muy poco Un poco Bastante Mucho }
$$

8. Enfriar el agua de una bañera hasta alcanzar la temperatura adecuada para darse un baño

$$
\text { Nada Muy poco Un poco Bastante Mucho }
$$

9. Diseñar y construir en el laboratorio o en el taller un circuito eléctrico simple con una bombilla

$$
\text { Nada Muy poco Un poco Bastante Mucho }
$$

10. Reemplazar la bombilla de un proyector

$$
\text { Nada Muy poco Un poco Bastante Mucho }
$$


11. Enfocar las lentes de tu cámara
Nada Muy poco Un poco Bastante Mucho

12. Cambiar el objetivo de un microscopio
Nada Muy poco Un poco Bastante Mucho

13. Usar un termómetro para registrar el punto de ebullición de una disolución

Nada Muy poco Un poco Bastante Mucho

14. Informarse acerca de los precios de las actividades extraescolares de tu centro escolar, para que tu opinión se tenga en cuenta en el próximo consejo escolar

Nada Muy poco Un poco Bastante Mucho

15. Tener un compañero en el laboratorio que te observa mientras realizas un experimento

Nada Muy poco Un poco Bastante Mucho

16. Visitar el museo de las Artes y las Ciencias y tener que explicar cómo se genera la energía nuclear a un niño de primaria
Nada Muy poco Un poco Bastante Mucho

17. Estudiar para un examen de Inglés, Historia o Filosofía

Nada Muy poco Un poco Bastante Mucho

18. Mezclar la cantidad adecuada de bicarbonato de sodio y agua para aplicar sobre una picadura de abeja

Nada Muy poco Un poco Bastante Mucho

19. Encender un camping gas (pequeño hornillo portátil) para preparar la comida durante una acampada

Nada Muy poco Un poco Bastante Mucho

20. Afinar una guitarra usando un piano u otro instrumento musical

Nada Muy poco Un poco Bastante Mucho

21. Llenar con la cantidad adecuada de aire las ruedas de tu bicicleta

Nada Muy poco Un poco Bastante Mucho

22. Memorizar una tabla de fechas históricas

Nada Muy poco Un poco Bastante Mucho

23. En un debate en una clase de Física, exponer un trabajo sobre física nuclear y tener que responder unas cuestiones que te preguntan

Nada Muy poco Un poco Bastante Mucho 
24. Leer en un idioma extranjero mientras un compañero te escucha

Nada Muy poco Un poco Bastante Mucho

25. Leer letreros de los edificios en un país extranjero

Nada Muy poco Un poco Bastante Mucho

26. Memorizar los nombres de los elementos de la tabla periódica

Nada Muy poco Un poco Bastante Mucho

27. Tocar un instrumento mientras el profesor de Música te escucha

Nada Muy poco Un poco Bastante Mucho

28. Leer la sección de 'Historia' de la revista Muy Interesante y que un amigo te pregunte tu opinión sobre lo leído

Nada Muy poco Un poco Bastante Mucho

29. En el laboratorio de Química, añadir pequeñas cantidades de un ácido a una disolución alcalina hasta neutralizarla

Nada Muy poco Un poco Bastante Mucho

30. Inflar de forma precisa un globo que será usado en un aparato de un experimento de física

Nada Muy poco Un poco Bastante Mucho

31. Encender un mechero Bunsen (que tiene una bombona pequeña de gas propano) para la realización de un experimento

Nada Muy poco Un poco Bastante Mucho

32. Informarse acerca de la energía nuclear, cuyo futuro es un tema de debate social, para adoptar una postura propia

Nada Muy poco Un poco Bastante Mucho

33. Usar un diapasón (instrumento metálico que vibra) en un experimento acústico

Nada Muy poco Un poco Bastante Mucho

34. Mezclar agua hirviendo y hielo para obtener una temperatura de $21^{\circ} \mathrm{C}$

Nada Muy poco Un poco Bastante Mucho

35. Estudiar un examen de Historia

Nada Muy poco Un poco Bastante Mucho

36. Realizar un experimento en el laboratorio mientras el profesor te observa

Nada Muy poco Un poco Bastante Mucho 
37. Realizar un experimento en el laboratorio mientras te observa el ayudante del profesor

Nada Muy poco Un poco Bastante Mucho

38. Enfocar un microscopio

Nada Muy poco Un poco Bastante Mucho

39. Utilizar un termómetro de cocina por primera vez y comprobar la temperatura de la comida periódicamente hasta que alcance la temperatura deseada

$$
\text { Nada Muy poco Un poco Bastante Mucho }
$$

40. Dibujar en la clase de arte mientras te observa el ayudante del profesor

$$
\text { Nada Muy poco Un poco Bastante Mucho }
$$

41. Leer la sección de 'Ciencia' de la revista Muy Interesante y que un amigo te pregunte tu opinión sobre lo leído

$$
\text { Nada Muy poco Un poco Bastante Mucho }
$$

42. Estudiar para un examen parcial de Química, Física o Biología

Nada Muy poco Un poco Bastante Mucho

43. Tener que explicar las cualidades artísticas del pop-art a un niño de primaria, durante una visita a un museo de Arte

$$
\text { Nada Muy poco Un poco Bastante Mucho }
$$

44. Preguntar una duda sobre literatura en clase de Castellano
Nada Muy poco Un poco Bastante Mucho 